\title{
Proportional Learning Vector Quantization
}

\author{
Rui-Ping LI $^{* 1}$ Masao MUKAIDONO*2
}

\section{Introduction}

Learning vector quantization(LVQ) is a clustering algorithm proposed by Kohonen[1] for organizing a large of unlabeled vectors into some clusters. Since the present principle of LVQ is related to the Perceptron idea, it is considered to be amenable to artificial neural networks. Although some good practical results have been obtained with it [1], this method suffers from a so-called initialization problem. Bezdek et al.[2] attempted to solve this problem by updating the winner and some of its metrical neighbors with each input. However, the another problem with this new strategy is how to choose an update neighborhood. More recently, Pal et al.[3] proposed a generalized learning vector quantization (GLVQ) algorithm, in which the learning rules were derived from an optimization problem. Unfortunately, we find that the GLVQ still does not solve the initialization problem completely. Especially when input vectors are normalized the GLVQ may fail to converge.

In this paper a proportional learning vector quantization(PLVQ) algorithm has been developed, which employs a fuzzy learning law to solve the normalization and initialization problems. The performance of the new algorithm has been compared to that of the learning vector quantization (LVQ) and generalized learning vector quantization (GLVQ) methods by two special examples. The results show that the presented method does not only avoid the initialization problem but also solve the normalization problem.

In the second section, we review briefly the LVQ and GLVQ methods. The third section contains a new algorithm that is called proportional learning vector quantization(PLVQ). Finally, in the fourth section the performance of the PLVQ algorithm is compared to that of the LVQ and GLVQ methods for two examples.

\footnotetext{
* I Dept. of Radiation Oncology, University of Rochester Medical Center

*2 Dept. of Computer Science, Meiji University 明治大学:
}

\section{Vector Quantization Algorithms}

The configuration of the LVQ clustering network is shown in Fig.1. $p$ represents the number of input variables. $X=\left\{\boldsymbol{x}_{1}, \boldsymbol{x}_{2}, \ldots, \boldsymbol{x}_{n}\right\}$ denotes a sample set that consists of $n$ data. $c$ represents the number of nodes (neurons) in the output layer. An output node corresponds to a cluster, and is connected to every one of input variables via pathways with weight one. Also, each output node is attached a prototype (center) vector, for example, $\boldsymbol{v}_{i}=\left(v_{i 1}\right.$, $\left.v_{i 2}, \ldots, v_{i p}\right)$ represents the prototype of cluster $i$. When the input $\boldsymbol{x}_{k}$ is presented to the network, the output $u_{i k}$ of node $i$ is calculated by the following formula

$$
\begin{aligned}
& \left\|\boldsymbol{x}_{k}-\boldsymbol{v}_{I}\right\|=\min _{i}\left\{\left\|\boldsymbol{x}_{k}-\boldsymbol{v}_{i}\right\|\right\} \\
& u_{i k}= \begin{cases}1, & \text { if } i=1 \\
0, & \text { otherwise }\end{cases}
\end{aligned}
$$

The standard form of the LVQ was written as [1] The LVQ algorithm :

1). Fix $2 \leq c \ll n, \varepsilon>0$ and the maximum number of iterations $T$.

2). Initialize $\left\{\boldsymbol{v}_{i}(0)\right\}$ and learning rate $\alpha_{0} \in[0,1]$.

3). For $t=1,2, \ldots, T$;

For $k=1,2, \ldots, n$;

a. Find the winner neuron $I$ using Eq. (1).

b. Update the prototype of the winner $I$

$$
\boldsymbol{v}_{i}(t)=\boldsymbol{v}_{I}(t-1)+\alpha_{0}(1-t / T)\left(\boldsymbol{x}_{k}-\boldsymbol{v}_{I}\right)
$$

c. Next $k$.

4). Calculate $E=\sum_{i=1}^{c} \sum_{j=1}^{p}\left|v_{i j}(t)-v_{i j}(t-1)\right|$.

5). IF $E<\varepsilon$ or $t>T$ stop ; ELSE next $t$.

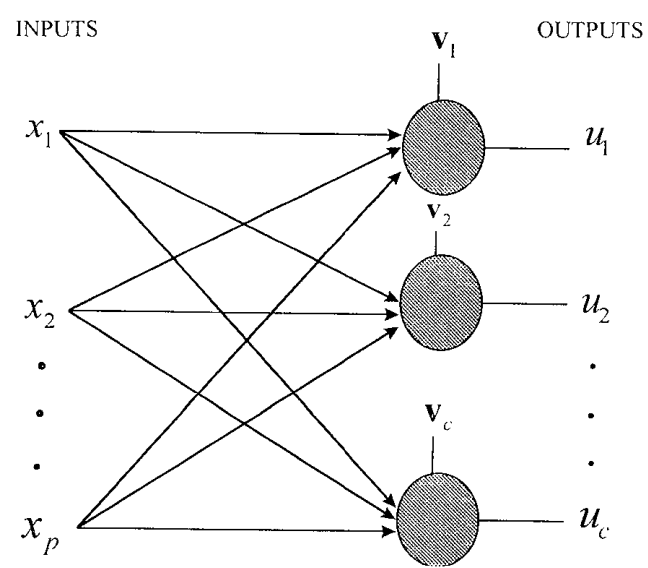

Fig.1. LVQ schematic diagram. 
In the above algorithm, Eq. (3) is called competitive learning law (CLL). As mentioned in the preceding section, the major problem with LVQ is that this technique depends upon the selection of initial prototypes $\left\{\boldsymbol{v}_{i}(0)\right\}$. It may converge to an undesired solution due to a special selection of initial values. In order to avoid this problem, N. R. Pal et al. proposed the GLVQ algorithm[3]. The updating rules of the GLVQ were derived from the following loss function which measures the locally weighted mismatch of $\boldsymbol{x}$ with respect to the winner $I$.

$$
L_{x}=\sum_{r=1}^{c} g_{I r}\left\|\boldsymbol{x}-\boldsymbol{v}_{r}\right\|^{2}
$$

where,

$$
g_{l r}=\left\{\begin{array}{l}
1, \quad \text { if } r=I \\
\frac{1}{\sum_{j=1}^{c}\left\|\boldsymbol{x}-\boldsymbol{v}_{j}\right\|^{2} .}
\end{array}\right. \text { otherwise }
$$

The purpose of the GLVQ is to find a set of prototypes $\left\{\boldsymbol{v}_{r}\right\}$, such that the locally weighted loss function $L_{x}$ defined with respect to the winner $\boldsymbol{v}_{I}$ is minimized over $X$. Differentiating $L_{x}$ with respect to $\boldsymbol{v}_{I}$, the following updating rules were obtained

$$
\left\{\begin{array}{l}
\Delta \boldsymbol{v}_{I}=\alpha_{t} \eta_{I}\left(\boldsymbol{x}-\boldsymbol{v}_{I}\right) \text { for winner } I \\
\Delta \boldsymbol{v}_{i}=\alpha_{t} \eta_{i}\left(\boldsymbol{x}-\boldsymbol{v}_{i}\right) \text { for each } i \neq I
\end{array}\right.
$$

where,

$$
\begin{aligned}
& \eta_{I}=\frac{D^{2}-D+\left\|\boldsymbol{x}-\boldsymbol{v}_{l}\right\|^{2}}{D^{2}}, \\
& \eta_{i}=\frac{\left\|\boldsymbol{x}-\boldsymbol{v}_{i}\right\|^{2}}{D^{2}} \text { and } D=\sum_{j=1}^{c}\left\|\boldsymbol{x}-\boldsymbol{v}_{i}\right\|^{2} .
\end{aligned}
$$

Although GLVQ improves the performance of the LVQ, GLVQ still exhibits one problem: When input vectors are normalized, this method may not converge because the $\eta_{I}$ and $\eta_{i}$ are too large.

\section{Proportional Learning Vector Quantization}

According to Hebb's learning postulate, we assume that the desired updating rules satisfy the following differential equations in continuous space

$$
\frac{\mathrm{d} \boldsymbol{v}_{i}}{d t}=\alpha_{t} \mathrm{u}_{i}(\boldsymbol{x})\left(\boldsymbol{x}-\boldsymbol{v}_{i}\right) \forall i
$$

or, in discrete domains we have

$$
\Delta \boldsymbol{v}_{i}=\alpha_{t} u_{i}(\boldsymbol{x})\left(\boldsymbol{x}-\boldsymbol{v}_{i}\right) \forall i
$$

Where $\alpha_{t}=\alpha_{0}(1-t / \mathrm{T})[1]$ and called temporal learning rate $; u_{i}(\boldsymbol{x}) \in[0,1]$ is the output value of the node $i$ when the input vector $\boldsymbol{x}$ is presented to the network. To find the mathematical expressions and physical meanings of the $u_{i}(v)$, consider a loss function with a fixed set of prototypes $\left\{\boldsymbol{v}_{i}\right\}$ as introduced in [5]

$$
L_{f}=\sum_{i=1}^{c} u_{i}(\boldsymbol{x})\left\|\boldsymbol{x}-\boldsymbol{v}_{i}\right\|^{2} .
$$

The physical meaning of minimizing the $L_{f}$ is : The more the input $\boldsymbol{x}$ is away from the prototype $\boldsymbol{v}_{i}$, the smaller the output $u_{i}(\boldsymbol{v})$ is. On the contrary, the closer the input $\boldsymbol{x}$ is to the prototype $\boldsymbol{v}_{i}$, the larger the output $u_{i}(\boldsymbol{x})$ is. According to the principle of maximum-entropy [6], the minimum cost problem having a loss function of the form (7) is converted into the following constrained optimization problem

$$
\begin{aligned}
& \text { maximize }\left(S(\boldsymbol{x})=-K \sum_{i=1}^{c} u_{i}(\boldsymbol{x}) \ln u_{i}(\boldsymbol{x})\right) \text {, } \\
& \text { subject to }\left(\text { a) } \sum_{i=1}^{c} u_{i}(\boldsymbol{x})\left\|\boldsymbol{x}-\boldsymbol{v}_{i}\right\|^{2}=\delta,\right. \\
& \text { (b) } \sum_{i=1}^{c} u_{i}(\boldsymbol{x})=1, \\
& \text { (c) }\left(\boldsymbol{v}_{i}\right) \text { are fixed. }
\end{aligned}
$$

Where $S(\boldsymbol{x})$ is called the entropy of fuzzy sets with respect to member $\boldsymbol{x}$, which is associated with the assignment of input $\boldsymbol{x}$ in $c$ fuzzy sets (clusters). In the condition(a), $\delta$ is a given and small positive number that represents the value of the loss function $L_{f}$. Using the Lagrangian multiplier method, we solve the Eq. (8) for $u_{i}(\boldsymbol{x})$ [5], and get

$$
u_{i}(\boldsymbol{x})=\frac{\exp \left[-\frac{\left\|\boldsymbol{x}-\boldsymbol{v}_{i}\right\|^{2}}{2 \sigma^{2}}\right]}{\sum_{j=1}^{c} \exp \left[-\frac{\left\|\boldsymbol{x}-\boldsymbol{v}_{j}\right\|^{2}}{2 \sigma^{2}}\right],} \forall \boldsymbol{i}
$$

where $\sigma$ is a Lagrangian multiplier. From the Eq. (9), we see that $u_{i}(\boldsymbol{x})$ is a Gaussian function similar to that of the radial basis-function (RBF) [4]. The physical meaning of the $u_{i}(\boldsymbol{x})$ represents the degree to which the input $\boldsymbol{x}$ matches the prototype vector $\boldsymbol{v}_{i}$. From a learning point of view, therefore, $u_{i}(\boldsymbol{x})$ is called spatial learning rate, and Eq. (6) is called fuzzy learning law (FLL). In the case of $u_{i}$ $(\boldsymbol{x})=\{0,1\}$ FLL reduces to CLL.

A schematic diagram of the proportional learning vector quantization (PLVQ) is shown in Fig.2. The smaller the $\sigma$ is, the less the fuzziness of clusters is. As $\sigma \rightarrow 0$, PLVQ reduces to LVQ. As $\sigma$ $\rightarrow \infty$, each of nodes has almost the same prototype vector, and thus each node losses the feature. Below, we summarize the PLVQ algorithm.

\section{The PLVQ Algorithm:}

1). Fix $2 \leq c \ll n, \varepsilon>0, \sigma>0$ and the maximum number of iterations $T$.

2). Initialize $\left\{\boldsymbol{v}_{i}(0)\right\}$ and learning rate $\alpha_{0} \in[0,1]$. 
Table 1. The data sets used in Example 1.

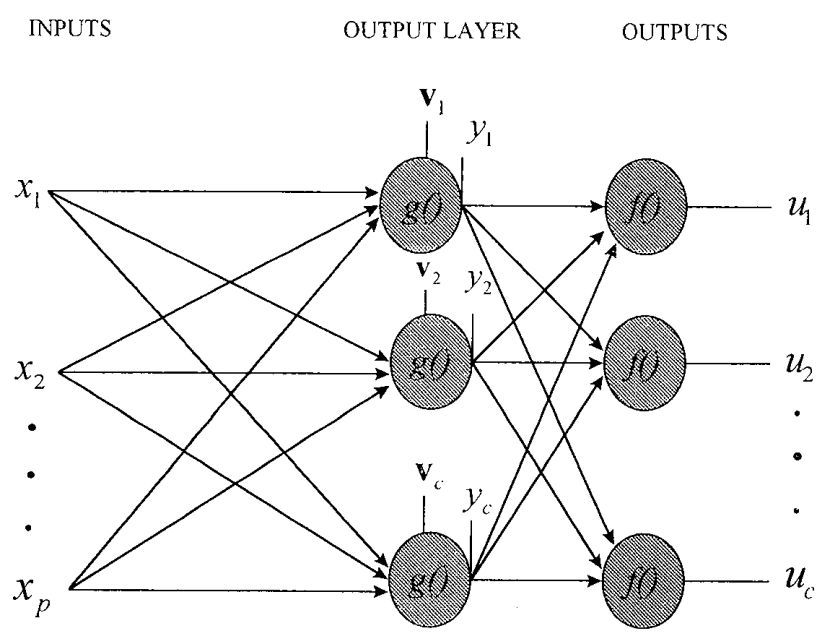

Fig.2. PLVQ schematic diagram. Here, $y_{i}=g\left(\mathbf{x} ; \mathbf{v}_{i}\right)=$ $\exp \left[-\left\|\mathbf{x}-\mathbf{v}_{i}\right\|^{2} /\left(2 \sigma^{2}\right)\right]$, and $u_{i}=f\left(s ; y_{i}\right)=y_{i} / s=y_{i} /$ $\sum_{j=1}^{c} y_{j}$

3). For $t=1,2, \ldots, T$;

For $k=1,2, \ldots, n$;

a. Calculate $\left\{u_{i}\left(\boldsymbol{x}_{k}\right)\right\}$ using Eq. (9).

b. Update $\left\{\boldsymbol{v}_{i}(t)\right\}$ using

$\boldsymbol{v}_{I}(t)=\boldsymbol{v}_{I}(t-1)+\alpha_{0}(1-t / T) u_{i}\left(\boldsymbol{x}_{k}\right)\left(\boldsymbol{x}_{k}-\boldsymbol{v}_{I}\right)$

c. Next $k$.

4). Calculate $E=\sum_{i=1}^{c} \sum_{j=1}^{p}\left|\boldsymbol{v}_{i j}(t)-v_{i j}(t-1)\right|$.

5). IF $E<\varepsilon$ or $t>T$ stop ; ELSE next $t$.

\section{Experimental Results}

Two examples have been selected to illustrate the performance of the PLVQ. The first example, a two-cluster problem which contains an artificial data sets of $n=3$ and $p=2$ will be used to make a comparison among the three methods. In the second example we select the IRIS data[7] to illustrate the importance of the normalization procedure in data analysis.

\section{Example 1: Two artificial data sets}

As shown in Table. 1, this example contains two data sets: Data Set 1 and Data Set 2. When the number of clusters is set to two $(c=2)$, the correct classification results of these two data sets are the same: cluster $1=\left\{\boldsymbol{x}_{1}, \boldsymbol{x}_{2}\right\}$, cluster $2=\left\{\boldsymbol{x}_{3}\right\}$. In this study, to investigate the effect of initial values $\left\{\boldsymbol{v}_{i}(0)\right\}$, we initialized the LVQ, GLVQ and PLVQ with three different strategies as follows:

I 1 : Using $n$ samples which distribute randomly $c$ clusters. In other words, first initialize $u_{1 i}\left(\boldsymbol{x}_{k}\right) \in$

\begin{tabular}{|c|c|}
\hline $\begin{array}{c}(a) \\
\text { Data Set } 1\end{array}$ & $\begin{array}{c}\text { (b) } \\
\text { Data Set 2 }\end{array}$ \\
\hline$x 1=(0.00,5.00)$ & $x 1=(0.00,0.50)$ \\
$x 2=(0.00,0.00)$ & $x 2=(0.00,0.00)$ \\
$x 3=(5.00,2.50)$ & $x 3=(0.50,0.25)$ \\
\hline
\end{tabular}

Table 2. Comparison of the classification results for Data Set 1.

\begin{tabular}{ccccc}
\hline \multirow{2}{*}{ Initialization } & $\alpha_{0}$ & \multicolumn{3}{c}{ Number of Errors } \\
\cline { 3 - 5 } & 0.2 & LVQ & GLVQ & PLVQ \\
\hline \multirow{2}{*}{ I1 } & 0.6 & 1 & 1 & 0 \\
& 0.9 & 0 & 0 & 0 \\
\hline \multirow{2}{*}{$12 \mathrm{a}$} & 0.2 & 1 & 1 & 0 \\
& 0.6 & 1 & 1 & 0 \\
& 0.9 & 0 & 0 & 0 \\
\hline \multirow{3}{*}{$12 \mathrm{~b}$} & 0.2 & 0 & 0 & 0 \\
& 0.6 & 0 & 0 & 0 \\
& 0.9 & 0 & 0 & 0 \\
\hline
\end{tabular}

$[0,1](i=1,2, \ldots, c)$ at random for each $k(k=$ $1,2, \ldots, n)$, and then calculate $\boldsymbol{v}_{i}=\sum_{k=1}^{n} u_{i}\left(\boldsymbol{x}_{k}\right) \boldsymbol{x}_{k}$ for each $i(i=1,2, \ldots, c)$.

I 2 a : Using $c$ special vectors, here $\boldsymbol{v}_{1}(0)=\boldsymbol{x}_{1}$ and $\boldsymbol{v}_{2}(0)=\boldsymbol{x}_{2}$.

I $2 \mathrm{~b}$ : Using $c$ special vectors, here and $\boldsymbol{v}_{1}(0)=\boldsymbol{x}_{1}$ and $\boldsymbol{v}_{2}(0)=\boldsymbol{x}_{3}$.

For each of the initialization strategies, the LVQ, GLVQ and PLVQ were run with three different initial values of $\alpha_{0}(0.2,0.6,0.9)$ for $\varepsilon=$ 0.0001 and $T=500$ iterations. First, the three methods were applied to Data Set 1(Table 1(a)). Table 2 displays the results. We see that GLVQ also depends upon the selection of initial values of $\left\{\boldsymbol{v}_{i}(0)\right\}$ and the selection of initial value of $\alpha_{0}$ like LVQ, whereas PLVQ works well for each of the three strategies.

Data Set 2 is a special data set, in which the value of each $x_{i j}(i=1,2 ; j=1,2)$ is less than or equal to one and is greater than or equal to zero. Obviously, Data Set 2 has the same structure as Data Set 1 does. However, in this case GLVQ never converges to the desired minimum for every one of the three strategies because the $\eta_{I}$ and $\eta_{I}$ are too large. 
It has been proved that the performance of neural networks can often be improved if the data set used to train is modified by removing insignificant characteristics. This is a normalization procedure. Normalization is an important and complicated topic. In general, there are two types of methods : offset normalization and deviation normalization[4]. Each of them can be further classified into vertical or horizontal. The offset normalization is used to remove the mean which is known to be irrelevant to the problem. The deviation normalization usually could overcome the scale problem of components of vectors.

IRIS data(Table 5) is that of $n=150, p=4$ and $c=3$. Each cluster contains 50 samples. Three clusters correspond to three subspecies of irises, respectively: Sestosa (1-50), Versicolor (51-100) and Virginica (101-150). This data has been used in many papers[3]. The LVQ, GLVQ and PLVQ methods were applied to IRIS data. For the original IRIS data, the numbers of mistakes are 17, 17 and 16, respectively. The characteristic of this data set is: each of feature variables has same scale and different offset. Therefore, we made vertical offset normalization for the original IRIS data using the following algorithm

Table 3. Comparison of the classification results for Data Set 2.

\begin{tabular}{ccccc}
\hline \multirow{2}{*}{ Initialization } & $\alpha_{i}$ & \multicolumn{3}{c}{ Number of Errors } \\
\cline { 3 - 5 } & 0.2 & LVQ & GLVQ & PLVQ \\
\hline \multirow{2}{*}{11} & 0.6 & 1 & 1 & 0 \\
& 0.9 & 0 & 1 & 0 \\
\hline \multirow{2}{*}{$12 \mathrm{a}$} & 0.2 & 1 & 1 & 0 \\
& 0.6 & 1 & 1 & 0 \\
& 0.9 & 0 & 1 & 0 \\
\hline \multirow{2}{*}{ I2b } & 0.2 & 0 & 1 & 0 \\
& 0.6 & 0 & 1 & 0 \\
& 0.9 & 0 & 1 & 0 \\
\hline
\end{tabular}

Table 4. Classification results for the normalized IRIS data. Here, $T$ denotes the number of iterations. In this case GLVQ does not work, whereas for PLVQ the number of mistakes is 6 that is much less than 17 in the case of using the original IRIS data.

\begin{tabular}{ccccccc}
\hline Method & $T$ & Initialization & $\alpha_{i 1}$ & $\varepsilon$ & $\sigma$ & $\begin{array}{c}\text { Number of } \\
\text { Errors }\end{array}$ \\
\hline GLVQ & 1000 & I1 & 0.1 & 2.0 & 0.1 & 43 \\
PLVQ & 1000 & I1 & 0.1 & 2.0 & 0.1 & 6 \\
\hline
\end{tabular}

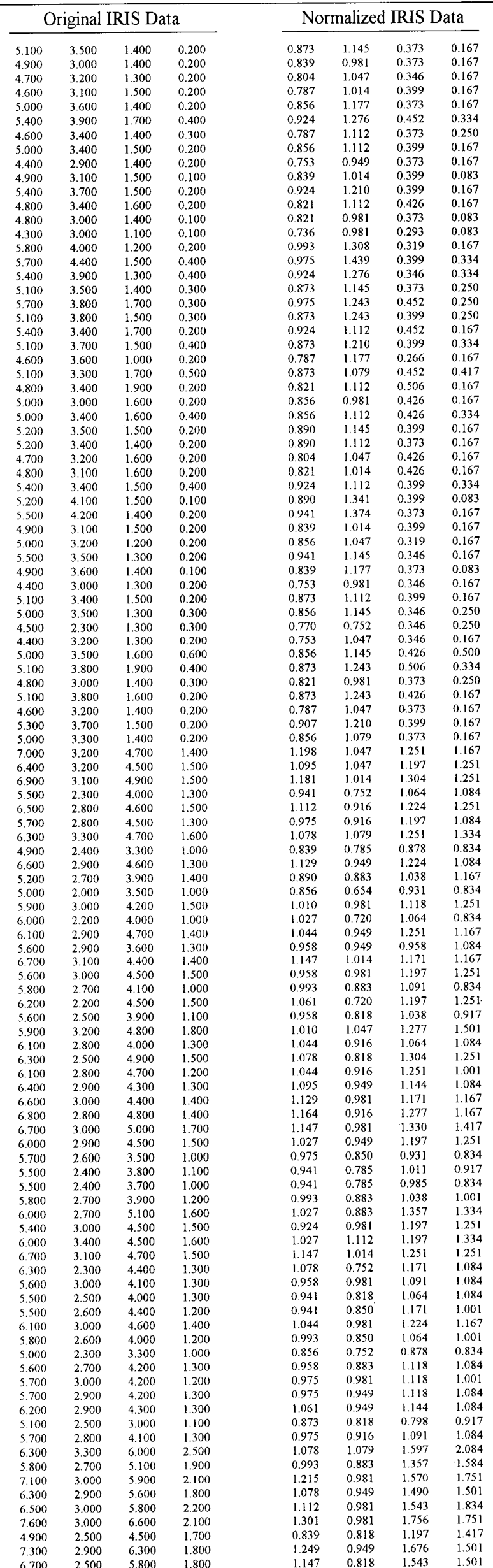




\begin{tabular}{|c|c|c|c|c|c|c|c|}
\hline 7.200 & 3.600 & 6.100 & 2.500 & 1.232 & 1.177 & 1.623 & 2.084 \\
\hline 6.500 & 3.200 & 5.100 & 2.000 & 1.112 & 1.047 & 1.357 & 1.668 \\
\hline 6.400 & 2.700 & 5.300 & 1.900 & 1.095 & 0.883 & 1.410 & 1.584 \\
\hline 6.800 & 3.000 & 5.500 & 2.100 & 1.364 & 0.981 & 1.464 & 1.751 \\
\hline 5.700 & 2.500 & 5.000 & 2.000 & 0.975 & 0.818 & 1.330 & 1.668 \\
\hline 5.800 & 2.800 & 5.100 & 2.400 & 0.993 & 0.916 & 1.357 & 2.001 \\
\hline 6.400 & 3.200 & 5.300 & 2.300 & 1.095 & 1.047 & 1.410 & 1.918 \\
\hline 6.500 & 3.000 & 5.500 & 1.800 & 1.112 & 0.981 & 1.464 & 1.501 \\
\hline 7.700 & 3.800 & 6.700 & 2.200 & 1.318 & 1.243 & 1.783 & 1.834 \\
\hline 7.700 & 2.600 & 6.900 & 2.300 & 1.318 & 0.850 & 1.836 & 1.918 \\
\hline 6.000 & 2.200 & 5.000 & 1.500 & 1.027 & 0.720 & 1.330 & 1.251 \\
\hline 6.900 & 3.200 & 5.700 & 2.300 & 1.181 & 1.047 & 1.517 & 1.918 \\
\hline 5.600 & 2.800 & 4.900 & 2.000 & 0.958 & 0.916 & 1.304 & 1.668 \\
\hline 7.700 & 2.800 & 6.700 & 2.000 & 1.318 & 0.916 & 1.783 & 1.668 \\
\hline 6.300 & 2.700 & 4.900 & 1.800 & 1.078 & 0.883 & 1.304 & 1.501 \\
\hline 6.700 & 3.300 & 5.700 & 2.100 & 1.147 & 1.079 & 1.517 & 1.751 \\
\hline 7.200 & 3.200 & 6.000 & 1.800 & 1.232 & 1.047 & 1.597 & 1.501 \\
\hline 6.200 & 2.800 & 4.800 & 1.800 & 1.061 & 0.916 & 1.277 & 1.501 \\
\hline 6.100 & 3.000 & 4.900 & 1.800 & 1.044 & 0.981 & 1.304 & 1.501 \\
\hline 6.400 & 2.800 & 5.600 & 2.100 & 1.095 & 0.916 & 1.490 & 1.751 \\
\hline 7.200 & 3.000 & 5.800 & 1.600 & 1.232 & 0.981 & 1.543 & 1.334 \\
\hline 7.400 & 2.800 & 6.100 & 1.900 & 1.266 & 0.916 & 1.623 & 1.584 \\
\hline 7.900 & 3.800 & 6.400 & 2.000 & 1.352 & 1.243 & 1.703 & 1.668 \\
\hline 6.400 & 2.800 & 5.600 & 2.200 & 1.095 & 0.916 & 1.490 & 1.834 \\
\hline 6.300 & 2.800 & 5.100 & 1.500 & 1.078 & 0.916 & 1.357 & 1.251 \\
\hline 6.100 & 2.600 & 5.600 & 1.400 & 1.044 & 0.850 & 1.490 & 1.167 \\
\hline 7.700 & 3.000 & 6.100 & 2.300 & 1.318 & 0.981 & 1.623 & 1.918 \\
\hline 6.300 & 3.400 & 5.600 & 2.400 & 1.078 & 1.112 & 1.490 & 2.001 \\
\hline 6.400 & 3.100 & 5.500 & 1.800 & 1.095 & 1.014 & 1.464 & 1.501 \\
\hline 6.000 & 3.000 & 4.800 & 1.800 & 1.027 & 0.981 & 1.277 & 1.501 \\
\hline 6.900 & 3.100 & 5.400 & 2.100 & 1.181 & 1.014 & 1.437 & 1.751 \\
\hline 6.700 & 3.100 & 5.600 & 2.400 & 1.147 & 1.014 & 1.490 & 2.001 \\
\hline 6.900 & 3.100 & 5.100 & 2.300 & 1.181 & 1.014 & 1.357 & 1.918 \\
\hline 5.800 & 2.700 & 5.100 & 1.900 & 0.993 & 0.883 & 1.357 & 1.584 \\
\hline 6.800 & 3.200 & 5.900 & 2.300 & 1.164 & 1.047 & 1.570 & 1.918 \\
\hline 6.700 & 3.300 & 5.700 & 2.500 & 1.147 & 1.079 & 1.517 & 2.084 \\
\hline 6.700 & 3.000 & 5.200 & 2.300 & 1.147 & 0.981 & 1.384 & 1.918 \\
\hline 6.300 & 2.500 & 5.000 & 1.900 & 1.078 & 0.818 & 1.330 & 1.584 \\
\hline 6.500 & 3.000 & 5.200 & 2.000 & 1.112 & 0.981 & 1.384 & 1.668 \\
\hline 6.200 & 3.400 & 5.400 & 2.300 & 1.061 & 1.112 & 1.437 & 1.918 \\
\hline 5.900 & 3.000 & 5.100 & 1.800 & 1.010 & 0.981 & 1.357 & 1.501 \\
\hline
\end{tabular}

For $p=1,2,3,4$

$$
\operatorname{sum}[p]=0 \text {; }
$$

For $k=1,2, \ldots, 150$

$$
\operatorname{sum}[p]=\operatorname{sum}[p]+\operatorname{data}[k][p] ;
$$

average $[p]=\operatorname{sum}[p] / 150$;

For $p=1,2,3,4$

For $k=1,2, \ldots, 150$

$$
\operatorname{data}[k][p]=\operatorname{data}[k][p] / \text { average }[p] ;
$$

Table 4 shows results of the GLVQ and PLVQ for the normalized IRIS data that is obtained by normalizing the original IRIS data using the above algorithm. In this case GLVQ does not work, whereas for PLVQ the number of mistakes is 6 that is much less than 16 in the case of using the original IRIS data. From this example, we see further the importance of the normalization procedure and the limitation of the GLVQ

\section{Conclusions}

We have developed the PLVQ technique based on the fuzzy learning law (FLL). The method does not only avoid the initialization problem in the LVQ, but also solve the normalization problem in the GLVQ. The properties of the PLVQ method suggest that it may be viable alternative to conventional learning vector quantization(LVQ).

\section{References}

[1]T. Kohonen, Self-organization and Associative Memory, Berlin : Springer-verlag, 1989.

[2] J.C. Bezdek, E.C. Tsao and N.R. Pal, “Fuzzy Kohonen clustering neural networks" in Proc. IEEE Int. Conf. Fuzzy Syst., Mar. 1992, San Diego, CA, pp. 10351041.

[3]N. R. Pal, J. C. Bezdek and E. C.-K. Tsao, "Generalized clustering networks and Kohonen's selforganizing scheme", IEEE Trans. On Neural Networks, vol. 4, pp. 549-557, 1993.

[4]P. D. Wasserman, Advanced Methods in Neural Computing, New York: Van Nostrand Reinhold, 1993.

[5]R.P. Li and M. Mukaidono, "A maximum-entropy approach to fuzzy clustering”, FUZZ-IEEE/IFES' 95 symposium, Yokohama, Japan, pp.2227-2233, 1995.

[6]E.T. Jaynes, "Information theory and statistical mechanics", Phys. Rev. vol. pp.106, 620-630, 1957 ; vol.108, pp.171-190, 1957.

[7]E. Anderson, "The IRISes of the Gaspe Peninsula", bulletin of the American IRIS society, vol. 59, pp. 2-5, 1939 .

[8]S. Lee and R.M. Kill, "A Gaussian potential function network with hierarchically self-organizing learn ing”, Neural Networks, 4(2), pp. 207-224, 1991.

（1997年10月 9H 受 付) （1998年. 8月12日 再受付）

[閣い命わせ先]

テ214-0038

川崎术多摩区東三田1-1-1

明治大学理工学部情報工学科

们殿政男

TEL : 044-934-7450

FAX : 044-934-7912

E-mail :masao@cs.meiji.ac.jp 
著 者紹介

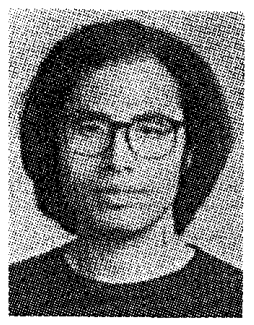

\section{Rui-Ping $\mathrm{Li}$}

Dept. of Radiation Oncology, University of Rochester Medical Center

He received the B.S. degree in physics from Nanking University, Nanking, China, in 1982, the M.S. degree in education from Tokyo Gakugei University, Tokyo, Japan, in 1992, the Ph.D. degree in electrical engineering from Meiji University, Tokyo, Japan, in 1995. During 1982-1988, he was an assistant professor in the Department of Physics, Huaqiao University, QUANZHOU, FUJIAN, China. Since 1988 he was came to Japan. He is currently a research staff of Radiation Oncology, Medical Center, University of Rochester. His research interests include pattern recognition, fuzzy logic, neural networks, and chaotic information processing.

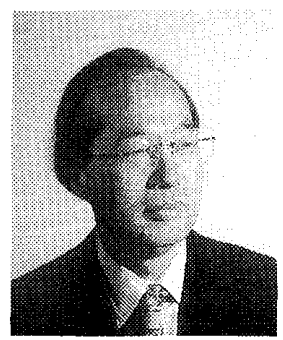

Masao MUKAIDONO

Dept. of Computer Science, Meiji University

He received the B.S. degree in electrical engineering from Meiji University, Kawasaki, Japan, in 1965, 1976 , and 1970 , respectivery. He is currently a Professor in the Department of Computer Science, School of Science and Technology, Meiji University. His main research interests are in multiple-valued logic, fuzzy logic, and its applications, fault-tolerant computing, fail-safe logic, and computer aided logic design. Dr.Mukaidono was president of Japan Society of Fuzzy Theory and Systems, and member of the IEEE Computer Society, the Information Processing Society of Japan, and Japanese Society of Artificial Intelligence.

\title{
Proportional Learning Vector Quantization
}

by

\section{Rui-Ping LI and Masao MUKAIDONO}

Abstract :

A proportional learning vector quantization(PLVQ) algorithm has been developed. The algorithm employs a fuzzy learning law to solve the normalization and initialization problems that are encountered in traditional learning vector quantization $(\mathrm{LVQ})$. The performance of the new algorithm has been compared to that of the learning vector quantization(LVQ) and generalized learning vector quantization(GLVQ) methods by two special examples. The results show that the presented method does not only avoid the initialization problem but also solve the normalization problem.

Keywords : Clustering Networks, Learning Vector Quantization, Fuzzy Logic

\author{
Contact Address : Masao MUKAIDONO \\ Dept. of Computer Science, Meiji University \\ 1-1-1 Higasi-Mita, Tama-ku, Kawasaki-shi, 214-0038 Japan \\ TEL : 044-934-7450 \\ FAX : 044-934-7912 \\ E-mail :masao@cs.meiji.ac.jp
}

